Original Research

\title{
Examining Sex Differences in Visual Reliance During Postural Control in Intercollegiate Athletes
}

\author{
Nicolette Ingel ${ }^{1}$, , Victoria Vice ${ }^{1}$, Courtney Dommer ${ }^{1}$, Jennifer Csonka ${ }^{2}$, Tara Moore ${ }^{3}$, Ariel Zaleski ${ }^{4}$, Carolyn Killelea ${ }^{5}$, \\ Mallory Faherty ${ }^{5}$, Jody Feld ${ }^{1}$, Timothy Sell ${ }^{5}$ \\ 1 Doctor of Physical Therapy Division, Department of Orthopaedic Surgery, Duke University, ${ }^{2}$ Department of Athletics, University of Pittsburgh, 3 \\ Department of Athletic Medicine, Duke University, ${ }^{4}$ Department of Athletics, Elon University, ${ }^{5}$ Michael W. Krzyzewski Human Performance \\ Laboratory, Department of Orthopaedic Surgery, Duke University \\ Keywords: vision, sex differences, postural stability \\ https://doi.org/10.26603/001c.28099
}

\section{International Journal of Sports Physical Therapy}

Vol. 16, Issue 5, 2021

\section{Background}

Risk factors for different sports injuries vary between sexes. Deficits in postural stability have been associated with several lower extremity injuries. The purpose of this study was to examine the differences in static postural stability between male and female intercollegiate athletes with and without visual information.

\section{Hypothesis}

There will be no difference in visual reliance between sexes during static postural stability.

\section{Study Design}

Cross-sectional Study

\section{Methods}

Static postural stability was assessed during a single session for football, soccer, basketball, and volleyball intercollegiate athletes (males, $n=135$, females, $n=51$ ) under eyes open (EO) and eyes closed (EC) conditions via performance of single limb stance on a force plate. Ground reaction force component data in all directions were quantified as a unitless composite score (COMP) where lower values indicated better postural stability. The absolute change and percentage change between EO and EC conditions were calculated for each sex. Two-sample Kolmogorov-Smirnov tests were used to compare differences between sexes.

\section{Results}

Males had greater EO COMP (males $=7.77 \pm 3.40$; females $=6.48 \pm 4.61 ; \mathrm{p}=0.038$; Cohen's $\mathrm{d}=0.343$ ) and EC COMP (males $=19.43 \pm 8.91$; females $14.66 \pm 6.65 ; \mathrm{p}=0.001$; Cohen’s $\mathrm{d}=0.571$ ) than females. A significant difference in absolute change from EO to EC was observed between sexes (males $=-11.65 \pm 7.05$; females $=-8.18 \pm 5.61 ; p=0.01$, Cohen's $\mathrm{d}=-0.520$ ) indicating that males had a greater change between conditions for the worse. There was no significant difference in percent change from EO to EC between sexes (males=159.2 \pm 90.7 ; females=156.7 $\pm 109.2 ; \mathrm{p}=0.39$; Cohen’s $\mathrm{d}=0.026$ ).

\section{Conclusions}

The observed differences between males and females in EO COMP, EC COMP, and absolute difference in COMP indicate that there is some factor that causes a difference in static postural stability between sexes. No difference in percent change between groups

\footnotetext{
Corresponding Author: Nicolette Ingel, PT, DPT Phone: 412-992-8842 Email:ningel20@gmail.com
} 
indicates that the difference in static postural stability between sexes may not be due to visual reliance. Female athletes may inherently have better postural stability than males, but both sexes were able to compensate for the loss of visual input.

\section{Level of Evidence}

\section{INTRODUCTION}

Musculoskeletal injuries are highly prevalent within collegiate athletics and demonstrate both short-term and longterm consequences. ${ }^{1}$ Recent data highlight that over one million injuries have occurred in student-athletes over a five-year period across 25 NCAA sports. ${ }^{1}$ Injury rates and risk for injury vary based on the sport being played, whether an athlete is at a practice or competition, and the sex of the athlete. ${ }^{1}$ In fact, sex differences in injury rates are very common. ${ }^{1-9}$ For example, the anterior cruciate ligament injury rate for intercollegiate female athletes is 2.5 times higher than intercollegiate male athletes for sex-comparable sports, ${ }^{9}$ as well as concussion rates being higher in female athletes for sex-comparable sports. ${ }^{10}$ Furthermore, research has demonstrated that females have a higher rate of stress fracture than males, and males are at greater risk of an acromioclavicular joint sprain than females. 3,7 Given the prevalence of sports injuries and the differences between sexes in injury rates, there appears to be a need to develop sex-specific injury prevention programs (IPP). To do so, sexspecific differences in risk factors for injuries must be identified.

Deficits in postural stability have been identified in several studies as a risk factor for lower extremity injuries such as anterior cruciate ligament and ankle injuries. 5,6,11 The results of these studies support the need to address postural stability as part of IPP. Previous authors have shown that males demonstrate significantly worse postural stability as compared to females; ${ }^{4,12}$ however, the evidence has not yet identified a clear reason(s) for this difference. Postural stability is a complex process that incorporates input from the visual, somatosensory, and vestibular systems and may also be affected by articular, ligamentous, and muscular structural differences. ${ }^{5}$ One potential reason for sex differences in postural stability may be differences in reliance on visual input. If this is true, the differences between males and females could contribute to differences in postural stability and thereby contribute to sex-specific differences in injury rates. The purpose of this study was to examine the differences in static postural stability between male and female intercollegiate athletes with and without visual information. It was hypothesized that there would be no betweensex differences in postural stability between varied conditions of visual input. The outcomes of this study could play a role in the creation of IPP for musculoskeletal injuries, specifically addressing whether postural stability training with altered visual input should be incorporated in sex-specific IPP.

\section{METHODS}

\section{PARTICIPANTS}

In this cross-sectional study, all participants were Division I NCAA football, soccer, basketball, and volleyball studentathletes (135 males and 51 females) between the ages of 18 and 25 , currently rostered and participating as a varsity level intercollegiate athlete and cleared by medical personnel (certified athletic trainer or team physician) to participate in the study. Subjects were excluded if they were not cleared by medical personnel to participate in full activity, if they had sustained a previous lower extremity surgery, or if they had sustained a lower extremity injury within the prior six months. All participants read and signed an informed consent form approved by the university where the data was collected.

\section{SINGLE LEG POSTURAL STABILITY ASSESSMENT}

\section{PROCEDURES}

Participants were tested in their respective athletic training rooms during a single session and were barefoot during testing. Controlled testing conditions included placing the force plate on a hard and level surface, eliminating noise during testing, removing distractions such as any movement of people in the area, and performing identical protocol regardless of physical space. Static postural stability was assessed $(1200 \mathrm{~Hz})$ while standing on a single force plate (Kistler 9286A, Kistler Corporation, Amherst, NY, USA) in two conditions: (1) eyes open (EO) and (2) eyes closed (EC). Testing for static postural stability began with participants assuming a single-leg stance on their dominant leg (self-reported preferred kicking foot), ${ }^{13}$ hands placed on hips; and with the non-stance leg flexed at the knee and hip in order to bring the foot to the height of the stance leg ankle, this same position was used during both EO and EC tests. For the EO condition, participants focused on a marker located approximately $6.10 \mathrm{~m}$ (20 feet) directly in front of the force plate on the wall at the height of the subject. For the EC condition, participants began identical to the EO condition and were directed to close their eyes once they were ready for data collection to begin. The protocol and data processing procedures employed for static postural stability allowed for touchdowns of the non-stance leg on the force plate, but participants were instructed to immediately return their non-stance leg back to the starting position if a touchdown occurred. A trial was discarded if the nonstance leg touched the stance leg or touched down on the ground off the force plate. The mean of three 10-second trials were collected for data analysis following the practice trials which consisted of a minimum of three repetitions but no more than five repetitions. The protocol used in the current study was based on a protocol previously described by 
Table 1. Descriptive data (Mean \pm SD) for all subjects, male subjects, and female subjects and independent $t-t e s t$ (p-values) between males and females.

\begin{tabular}{lcccc}
\hline Variable & All & Males & Females & p-value \\
\hline Age $(\mathrm{yrs})$ & $19.5 \pm 1.3$ & $19.6 \pm 1.3$ & $19.3 \pm 1.2$ & 0.103 \\
Height $(\mathrm{cm})$ & $181.6 \pm 10.0$ & $184.9 \pm 8.4$ & $172.9 \pm 8.7$ & $<0.001$ \\
Weight $(\mathrm{kg})$ & $79.3 \pm 14.6$ & $83.7 \pm 13.6$ & $67.6 \pm 10.1$ & $<0.001$ \\
\hline
\end{tabular}

Goldie et al. with demonstrated reliability and validity. ${ }^{14-16}$

\section{INSTRUMENTATION}

Force plate data were passed through an amplifier and an analog to digital board (DT3010, [Digital Translation, Marlboro, MA, USA]) to a personal computer for additional signal and data processing.

\section{DATA PROCESSING}

A MATLAB (v7.0.4; Natick, MA, USA) script file was written to process the data. All force plate data were initially filtered with a dual pass 4 th order low pass Butterworth filter with the cutoff frequency set at $20 \mathrm{~Hz}$. The standard deviation of each of the ground reaction force components (anterior-posterior, medial-lateral, and vertical) was calculated during the ten-second trial to derive the variables for statistical analysis which included the standard deviation of the anterior-posterior, medial-lateral, and vertical ground reaction forces. ${ }^{15}$ Lower values for all variables indicate better scores. For example, if an individual remained absolutely still, all of these variables would be zero. The more movement, the higher the scores. A unitless composite score (COMP) for EO and EC conditions was calculated by summing the standard deviation scores in each direction. The absolute change (EO-EC COMP, mean and standard deviation) was calculated as the difference between EO and EC conditions for each sex. The percent change ((EC-EO)/EO * 100 , mean and standard deviation) for each sex was also calculated between EO and EC conditions.

\section{STATISTICAL ANALYSIS}

Statistical analysis was completed using STATA (v14.2, College Station, TX, USA). The means and standard deviations of COMP scores were calculated for both males and females under EO and EC conditions. Two-sample KolmogorovSmirnov tests of the equality of distributions were performed for each of the postural stability variables since a Shapiro-Wilk test for normality demonstrated that each of the variables were not normally distributed. The level of statistical significance was set at $\mathrm{p}<0.05$ a priori. Effect sizes (Cohen's $d$ ) and 95\% confidence intervals were also calculated.

\section{RESULTS}

A demographic breakdown of participants is presented in
Table 1, which includes 135 males and 51 female participants. A significant difference was found for both height and weight between sexes. The means and standard deviations of the eyes open composite score (EO COMP), eyes closed composite score (EC COMP), absolute change, and percent change for both males and females are presented in Table 2. The two-sample Kolmogorov-Smirnov tests comparing male and female athletes and effect sizes are also reported in Table 2 for each variable. Male athletes had significantly worse static postural stability COMP scores than female athletes under both EO and EC conditions. The absolute change between EO and EC conditions was significantly different between groups with male athletes demonstrating a significantly greater change in scores $(-11.65 \pm 7.05)$ than female athletes $(-8.18 \pm 5.61 ; p=0.01$, Cohen's $d=-0.520)$. The percent change from the EO to EC conditions between groups was not significantly different $(p=0.39)$.

\section{DISCUSSION}

Females demonstrated significantly better overall single limb postural stability than males for both EO and EC conditions. There was no significant difference between sexes in percent change (from EO to EC conditions), which indicates that the observed difference in postural stability between males and females may not be due to a difference in the use of visual input. The hypothesis that there would be no meaningful between-sex difference in static postural stability in response to changes in visual input was supported. These data indicate that there may be a factor other than the use of visual input that contributes to the difference in static postural stability between males and females such as differences in anthropometric variables (e.g., height, weight), joint laxity, and other unknown factors. The observed differences between males and females may indicate that IPP should be adjusted according to sex to account for these postural stability differences, but it may not be necessary to have sex-specific adjustments in availability of visual cues. It is important to note that postural stability is important for injury prevention in both sexes. ${ }^{5,6}$ While females have a greater risk for LE injury, some males do remain at risk for lower extremity injury. Given the demonstrated differences between males and females, the results of the current study suggest that individuals designing comprehensive IPP for male athletes may want to consider additional focus/time on postural stability training. ${ }^{17}$

In order to further investigate differences in postural stability between sexes, the complex nature of postural sta- 
Table 2. Eyes open composite, eyes closed composite, absolute change, and percent change scores based on sex.

\begin{tabular}{|c|c|c|c|c|c|c|c|}
\hline \multirow{2}{*}{ Variable } & \multicolumn{2}{|c|}{ Males $(n=135)$} & \multicolumn{2}{|c|}{ Females $(n=51)$} & \multirow{2}{*}{$\begin{array}{c}\mathrm{p}- \\
\text { value }\end{array}$} & \multirow{2}{*}{$\begin{array}{c}\text { Effect } \\
\text { size } \\
\text { (Cohen's } \\
\text { d) }\end{array}$} & \multirow{2}{*}{$\begin{array}{l}\text { 95\% Confidence } \\
\text { Interval }\end{array}$} \\
\hline & $\begin{array}{l}\text { Mean } \pm \\
\text { SD }\end{array}$ & Range & $\begin{array}{l}\text { Mean } \pm \\
\text { SD }\end{array}$ & Range & & & \\
\hline $\begin{array}{l}\text { Eyes Open } \\
\text { Composite }\end{array}$ & $\begin{array}{c}7.77 \pm \\
3.40\end{array}$ & $\begin{array}{l}3.28 \text { to } \\
26.20\end{array}$ & $\begin{array}{c}6.48 \pm \\
4.61\end{array}$ & $\begin{array}{l}2.96 \text { to } \\
27.77\end{array}$ & 0.038 & 0.343 & 0.018 to 0.666 \\
\hline $\begin{array}{l}\text { Eyes Closed } \\
\text { Composite }\end{array}$ & $\begin{array}{c}19.43 \pm \\
8.91\end{array}$ & $\begin{array}{l}6.49 \text { to } \\
75.52\end{array}$ & $\begin{array}{c}14.66 \pm \\
6.65\end{array}$ & $\begin{array}{l}4.72 \text { to } \\
33.53\end{array}$ & 0.001 & 0.571 & 0.242 to 0.897 \\
\hline $\begin{array}{l}\text { Absolute Change } \\
\text { (EO-EC) }\end{array}$ & $\begin{array}{c}-11.65 \pm \\
7.05\end{array}$ & $\begin{array}{c}-52.75 \text { to } \\
-1.80\end{array}$ & $\begin{array}{c}-8.18 \pm \\
5.61\end{array}$ & $\begin{array}{l}-28.98 \\
\text { to } 2.21\end{array}$ & 0.002 & -0.520 & -0.846 to -0.193 \\
\hline $\begin{array}{l}\text { Percent Change of } \\
\text { EO to EC }\end{array}$ & $\begin{array}{l}159.2 \pm \\
90.7\end{array}$ & $\begin{array}{l}27.5 \text { to } \\
639.7\end{array}$ & $\begin{array}{c}156.7 \pm \\
109.2\end{array}$ & $\begin{array}{l}3.9 \text { to } \\
636.7\end{array}$ & 0.875 & 0.026 & -0.296 to 0.348 \\
\hline
\end{tabular}

SD: Standard deviation

EO: Eyes open

EC: Eyes closed

bility should be addressed further by incorporating the assessment of all of the systems used to maintain postural stability including the visual, somatosensory, and vestibular systems. ${ }^{18,19}$ One or a combination of these systems may be inherently different between sexes and contribute to the established differences in postural stability between sexes. The results of the current study indicate that the differences in postural stability between sexes may not be solely due to differences in access to visual cues. When visual cues are removed, the somatosensory and vestibular systems are used to maintain postural stability. ${ }^{18,19}$ Males and females may incorporate somatosensory information or vestibular information differently for postural stability, or there may be other intrinsic factors (e.g., height, weight, etc.) that also contribute to the differences in postural stability between sexes. Further research should be performed to explore any sex-specific differences in the use of visual, vestibular, and somatosensory systems to maintain postural stability in collegiate athletes.

The current study has some limitations. First, without testing conditions involving the presence and absence of visual, somatosensory, and vestibular input, the conclusion cannot be drawn that any observed differences were due to only visual cues. Another limitation of this study is that there were unequal groups with males having 135 participants and females having 51 participants. Anthropometric differences between groups were also not taken into consideration and could play a role in postural stability differences as discussed above. Lastly, the study consisted only of intercollegiate athletes with an average age of 19.5 years who played football, soccer, basketball, or volleyball. Therefore, the results of this study cannot be generalized to other age groups, sports, or competition levels.

\section{CONCLUSION}

The observed differences between males and females in EO COMP, EC COMP, and absolute difference in COMP indicate that there is some factor that causes a difference in static postural stability between males and females. Postural stability is important for injury prevention in both sexes. The results of the current study suggest that individuals designing comprehensive IPP for males may want to consider additional focus/time on postural stability training as compared to those for females. No observed difference in percent change between groups indicates that the difference in static postural stability between males and females is likely not due to availability of visual information alone. Further research should be performed to determine if it is necessary for sex-specific IPP to address visual input during postural stability exercises. The differences observed between sexes in this study are likely due to some other inherent differences between the two groups included in this study or other factors not tested in the current study.

\section{CONFLICTS OF INTEREST}

We have no conflicts of interest to disclose.

Submitted: April 30, 2021 CDT, Accepted: August 23, 2021 CDT 


\section{REFERENCES}

1. Kerr ZY, Marshall SW, Dompier TP, Corlette J, Klossner DA, Gilchrist J. College sports-related injuries - United States, 2009-10 through 2013-14 academic years. MMWR Morb Mortal Wkly Rep. 2015;64(48):1130-1336.

2. Abt JP, Sell TC, Laudner KG, et al. Neuromuscular and biomechanical characteristics do not vary across the menstrual cycle. Knee Surg Sports Traumatol Arthrosc. 2007;15(7):901-907. doi:10.1007/s00167-00 7-0302-3

3. Hibberd EE, Kerr ZY, Roos KG, Djoko A, Dompier TP. Epidemiology of acromioclavicular joint sprains in 25 National Collegiate Athletic Association sports. Am J Sports Med. 2016;44(10):2667-2674. doi:10.1177/ 0363546516643721

4. Howell DR, Hanson E, Sugimoto D, Stracciolini A, Meehan WP. Assessment of the postural stability of female and male athletes. Clin J Sport Med. 2017;27(5):444-449. doi:10.1097/jsm.0000000000000 374

5. Murphy DF. Risk factors for lower extremity injury: A review of the literature. $\mathrm{Br} J$ Sports $\mathrm{Med}$. 2003;37(1):13-29. doi:10.1136/bjsm.37.1.13

6. Oshima T, Nakase J, Takata Y, Numata H, Tsuchiya $\mathrm{H}$. Poor static balance is a novel risk factor for noncontact anterior cruciate ligament injury. Orthop J Sports Med. 2015;3(7_suppl2). doi:10.1177/232596711 $\underline{5 \mathrm{~s} 00110}$

7. Rizzone KH, Ackerman KE, Roos KG, Dompier TP, Kerr ZY. The epidemiology of stress fractures in collegiate student-athletes, 2004-2005 Through 2013-2014 Academic Years. J Athl Train. 2017;52(10):966-975. doi:10.4085/1062-6050-52.8.01

8. Song K, Rhodes E, Wikstrom EA. Balance training does not alter reliance on visual information during static stance in those with chronic ankle instability: A Systematic Review with Meta-Analysis. Sports Med. 2017;48(4):893-905. doi:10.1007/s40279-017-0850-8

9. Stanley LE, Kerr ZY, Dompier TP, Padua DA. Sex differences in the incidence of anterior cruciate ligament, medial collateral ligament, and meniscal injuries in collegiate and high school sports. Am J Sports Med. 2016;44(6):1565-1572. doi:10.1177/03635 46516630927

10. Dick RW. Is there a gender difference in concussion incidence and outcomes? Br J Sports Med. 2009;43(Suppl I):i46-i50. doi:10.1136/bjsm.2009.0581 $\underline{72}$
11. Wikstrom EA, Song K, Pietrosimone BG, Blackburn JT, Padua DA. Visual utilization during postural control in anterior cruciate ligamentdeficient and -reconstructed patients: Systematic Reviews and Meta-Analyses. Arch Phys Med Rehabil. 2017;98(10):2052-2065. doi:10.1016/j.apmr.2017.04.0 10

12. Sell TC, Lovalekar MT, Nagai T, Wirt MD, Abt JP, Lephart SM. Gender differences in static and dynamic postural stability of soldiers in the Army's 101st Airborne Division (Air Assault). J Sport Rehabil. 2018;27(2):126-131. doi:10.1123/jsr.2016-0131

13. van Melick N, Meddeler BM, Hoogeboom TJ, Nijhuis-van der Sanden MWG, van Cingel REH. How to determine leg dominance: The agreement between self-reported and observed performance in healthy adults. PLoS ONE. 2017;12(12):e0189876. doi:10.137 1/journal.pone.0189876

14. Goldie PA, Bach TM, Evans OM. Force platform measures for evaluating postural control: Reliability and validity. Arch Phys Med Rehabil. 1989;70:510-517.

15. Goldie PA, Evans OM, Bach TM. Steadiness in one-legged stance: Development of a reliable forceplatform testing procedure. Arch Phys Med Rehabil. 1992;73(4):348-354. doi:10.1016/0003-9993(92)9000 $\underline{8-\mathrm{k}}$

16. Sell TC. An examination, correlation, and comparison of static and dynamic measures of postural stability in healthy, physically active adults. Phys Ther Sport. 2012;13(2):80-86. doi:10.1016/j.pts p.2011.06.006

17. McKeon PO, Hertel J. Systematic review of postural control and lateral ankle instability, part II: Is balance training clinically Effective? J Athl Train. 2008;43(3):305-315. doi:10.4085/1062-6050-43.3.305

18. Maurer C, Mergner T, Bolha B, Hlavacka F. Vestibular, visual, and somatosensory contributions to human control of upright stance. Neurosci Lett. 2000;281(2-3):99-102. doi:10.1016/s0304-3940(00)00 $\underline{814-4}$

19. Nashner LM, Shupert CL, Horak FB, Black FO. Organization of posture controls: An analysis of sensory and mechanical constraints. Prog Brain Res. 1989:411-418. doi:10.1016/s0079-6123(08)62237-2 